Electronic Supplementary Material (ESI) for New Journal of Chemistry.

This journal is (C) The Royal Society of Chemistry and the Centre National de la Recherche Scientifique 2018

\title{
Mono BN-Substituted Analogues of Naphthalene: A Theoretical Analysis of the Effect of BN Position on Stability, Aromaticity and Frontier Orbital Energies
}

Milovan Stojanovića and Marija Baranac-Stojanović*b

a University of Belgrade, Institute of Chemistry, Technology and Metallurgy - Center for Chemistry, Njegoševa 12,

P.O.Box 473, 11000 Belgrade, Serbia

${ }^{\mathrm{b}}$ University of Belgrade - Faculty of Chemistry, Studentski trg 12-16, P.O.Box 158, 11000 Belgrade, Serbia

\section{Supplementary Information}




\section{Absolute energies (atomic units) and $x, y, z$ coordinates ( $(̊)$}

\section{of the optimized structures}

\begin{tabular}{|c|c|c|c|c|c|}
\hline \multirow{2}{*}{$\begin{array}{l}\text { Center } \\
\text { Number }\end{array}$} & \multirow{2}{*}{$\begin{array}{l}\text { Atomic } \\
\text { Number }\end{array}$} & \multirow{2}{*}{$\begin{array}{l}\text { Atomic } \\
\text { Type }\end{array}$} & \multicolumn{3}{|c|}{ Coordinates (Angstroms) } \\
\hline & & & $\mathrm{X}$ & $Y$ & Z \\
\hline 1 & 6 & 0 & 0.000000 & 2.430634 & 0.707529 \\
\hline 2 & 6 & 0 & 0.000000 & 1.243886 & 1.400697 \\
\hline 3 & 6 & 0 & 0.000000 & 0.000000 & 0.715970 \\
\hline 4 & 6 & 0 & 0.000000 & 0.000000 & -0.715970 \\
\hline 5 & 6 & 0 & 0.000000 & 1.243886 & -1.400697 \\
\hline 6 & 6 & 0 & 0.000000 & 2.430634 & -0.707529 \\
\hline 7 & 1 & 0 & 0.000000 & -1.243119 & 2.485975 \\
\hline 8 & 1 & 0 & 0.000000 & 3.373020 & 1.243931 \\
\hline 9 & 1 & 0 & 0.000000 & 1.243119 & 2.485975 \\
\hline 10 & 6 & 0 & 0.000000 & -1.243886 & 1.400697 \\
\hline 11 & 6 & 0 & 0.000000 & -1.243886 & -1.400697 \\
\hline 12 & 1 & 0 & 0.000000 & 1.243119 & -2.485975 \\
\hline 13 & 1 & 0 & 0.000000 & 3.373020 & -1.243931 \\
\hline 14 & 6 & 0 & 0.000000 & -2.430634 & -0.707529 \\
\hline 15 & 6 & 0 & 0.000000 & -2.430634 & 0.707529 \\
\hline 16 & 1 & 0 & 0.000000 & -1.243119 & -2.485975 \\
\hline 17 & 1 & 0 & 0.000000 & -3.373020 & -1.243931 \\
\hline 18 & 1 & 0 & 0.000000 & -3.373020 & 1.243931 \\
\hline
\end{tabular}

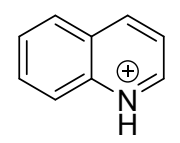

$E=-402.4085276 \mathrm{a} \cdot \mathrm{u}$.

\begin{tabular}{|c|c|c|c|c|c|}
\hline \multirow{2}{*}{$\begin{array}{l}\text { Center } \\
\text { Number }\end{array}$} & \multirow{2}{*}{$\begin{array}{l}\text { Atomic } \\
\text { Number }\end{array}$} & \multirow{2}{*}{$\begin{array}{c}\text { Atomic } \\
\text { Type }\end{array}$} & \multicolumn{3}{|c|}{ Coordinates (Angstroms) } \\
\hline & & & $\mathrm{x}$ & $Y$ & Z \\
\hline 1 & 6 & 0 & 2.391139 & 0.786789 & 0.000000 \\
\hline 2 & 6 & 0 & 1.179382 & 1.442393 & 0.000000 \\
\hline 3 & 6 & 0 & 0.000000 & 0.679293 & 0.000000 \\
\hline 4 & 6 & 0 & 0.042125 & -0.746933 & 0.000000 \\
\hline 5 & 6 & 0 & 1.311597 & -1.380460 & 0.000000 \\
\hline 6 & 6 & 0 & 2.459501 & -0.626439 & 0.000000 \\
\hline 7 & 1 & 0 & -1.272344 & 2.293460 & 0.000000 \\
\hline 8 & 1 & 0 & 3.307905 & 1.364169 & 0.000000 \\
\hline 9 & 1 & 0 & 1.132666 & 2.525766 & 0.000000 \\
\hline 10 & 6 & 0 & -1.179324 & -1.454528 & 0.000000 \\
\hline 11 & 1 & 0 & 1.357637 & -2.463025 & 0.000000 \\
\hline 12 & 1 & 0 & 3.427714 & -1.111513 & 0.000000 \\
\hline 13 & 6 & 0 & -2.391339 & -0.786896 & 0.000000 \\
\hline 14 & 6 & 0 & -2.394102 & 0.604154 & 0.000000 \\
\hline 15 & 1 & 0 & -1.158707 & -2.538941 & 0.000000 \\
\hline
\end{tabular}




$\begin{array}{rrrrrr}16 & 1 & 0 & -3.333039 & -1.318262 & 0.000000 \\ 17 & 1 & 0 & -3.301088 & 1.194602 & 0.000000 \\ 18 & 7 & 0 & -1.239231 & 1.278501 & 0.000000 \\ - & --1.0-----1\end{array}$<smiles></smiles>

$E=-402.4077095 \mathrm{a} . \mathrm{u}$.

\begin{tabular}{|c|c|c|c|c|c|}
\hline \multirow{2}{*}{$\begin{array}{l}\text { Center } \\
\text { Number }\end{array}$} & \multirow{2}{*}{$\begin{array}{l}\text { Atomic } \\
\text { Number }\end{array}$} & \multirow{2}{*}{$\begin{array}{l}\text { Atomic } \\
\text { Type }\end{array}$} & \multicolumn{3}{|c|}{ Coordinates (Angstroms) } \\
\hline & & & $\mathrm{X}$ & $\mathrm{Y}$ & Z \\
\hline 1 & 6 & 0 & 2.408292 & 0.834933 & 0.000000 \\
\hline 2 & 6 & 0 & 1.193174 & 1.471815 & 0.000000 \\
\hline 3 & 6 & 0 & 0.000000 & 0.700867 & 0.000000 \\
\hline 4 & 6 & 0 & 0.068367 & -0.735500 & 0.000000 \\
\hline 5 & 6 & 0 & 1.337517 & -1.353148 & 0.000000 \\
\hline 6 & 6 & 0 & 2.477731 & -0.580300 & 0.000000 \\
\hline 7 & 1 & 0 & -1.397479 & 2.376630 & 0.000000 \\
\hline 8 & 1 & 0 & 3.324363 & 1.412610 & 0.000000 \\
\hline 9 & 1 & 0 & 1.131987 & 2.553874 & 0.000000 \\
\hline 10 & 6 & 0 & -1.262263 & 1.302283 & 0.000000 \\
\hline 11 & 6 & 0 & -1.148310 & -1.468238 & 0.000000 \\
\hline 12 & 1 & 0 & 1.404017 & -2.434537 & 0.000000 \\
\hline 13 & 1 & 0 & 3.449486 & -1.059915 & 0.000000 \\
\hline 14 & 6 & 0 & -2.346766 & -0.819161 & 0.000000 \\
\hline 15 & 1 & 0 & -1.133262 & -2.550784 & 0.000000 \\
\hline 16 & 1 & 0 & -3.307619 & -1.313058 & 0.000000 \\
\hline 17 & 1 & 0 & -3.268270 & 1.019624 & 0.000000 \\
\hline 18 & 7 & 0 & -2.367098 & 0.553463 & 0.000000 \\
\hline
\end{tabular}

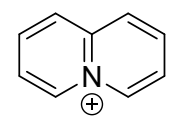

$E=-402.3934158$ a.u.

\begin{tabular}{|c|c|c|c|c|c|}
\hline \multirow{2}{*}{$\begin{array}{l}\text { Center } \\
\text { Number }\end{array}$} & \multirow{2}{*}{$\begin{array}{l}\text { Atomic } \\
\text { Number }\end{array}$} & \multirow{2}{*}{$\begin{array}{c}\text { Atomic } \\
\text { Type }\end{array}$} & \multicolumn{3}{|c|}{ Coordinates (Angstroms) } \\
\hline & & & $\mathrm{X}$ & $\mathrm{Y}$ & Z \\
\hline 1 & 6 & 0 & 0.000000 & -2.390025 & 0.711528 \\
\hline 2 & 6 & 0 & 0.000000 & -1.195409 & 1.362326 \\
\hline 3 & 6 & 0 & 0.000000 & 0.000000 & -0.728168 \\
\hline 4 & 6 & 0 & 0.000000 & -1.243741 & -1.399443 \\
\hline 5 & 6 & 0 & 0.000000 & -2.422758 & -0.702010 \\
\hline 6 & 1 & 0 & 0.000000 & 1.103597 & 2.439164 \\
\hline 7 & 1 & 0 & 0.000000 & -3.302854 & 1.292426 \\
\hline 8 & 1 & 0 & 0.000000 & -1.103597 & 2.439164 \\
\hline 9 & 6 & 0 & 0.000000 & 1.195409 & 1.362326 \\
\hline 10 & 6 & 0 & 0.000000 & 1.243741 & -1.399443 \\
\hline 11 & 1 & 0 & 0.000000 & -1.229079 & -2.481753 \\
\hline 12 & 1 & 0 & 0.000000 & -3.371456 & -1.223666 \\
\hline 13 & 6 & 0 & 0.000000 & 2.422758 & -0.702010 \\
\hline 14 & 6 & 0 & 0.000000 & 2.390025 & 0.711528 \\
\hline
\end{tabular}




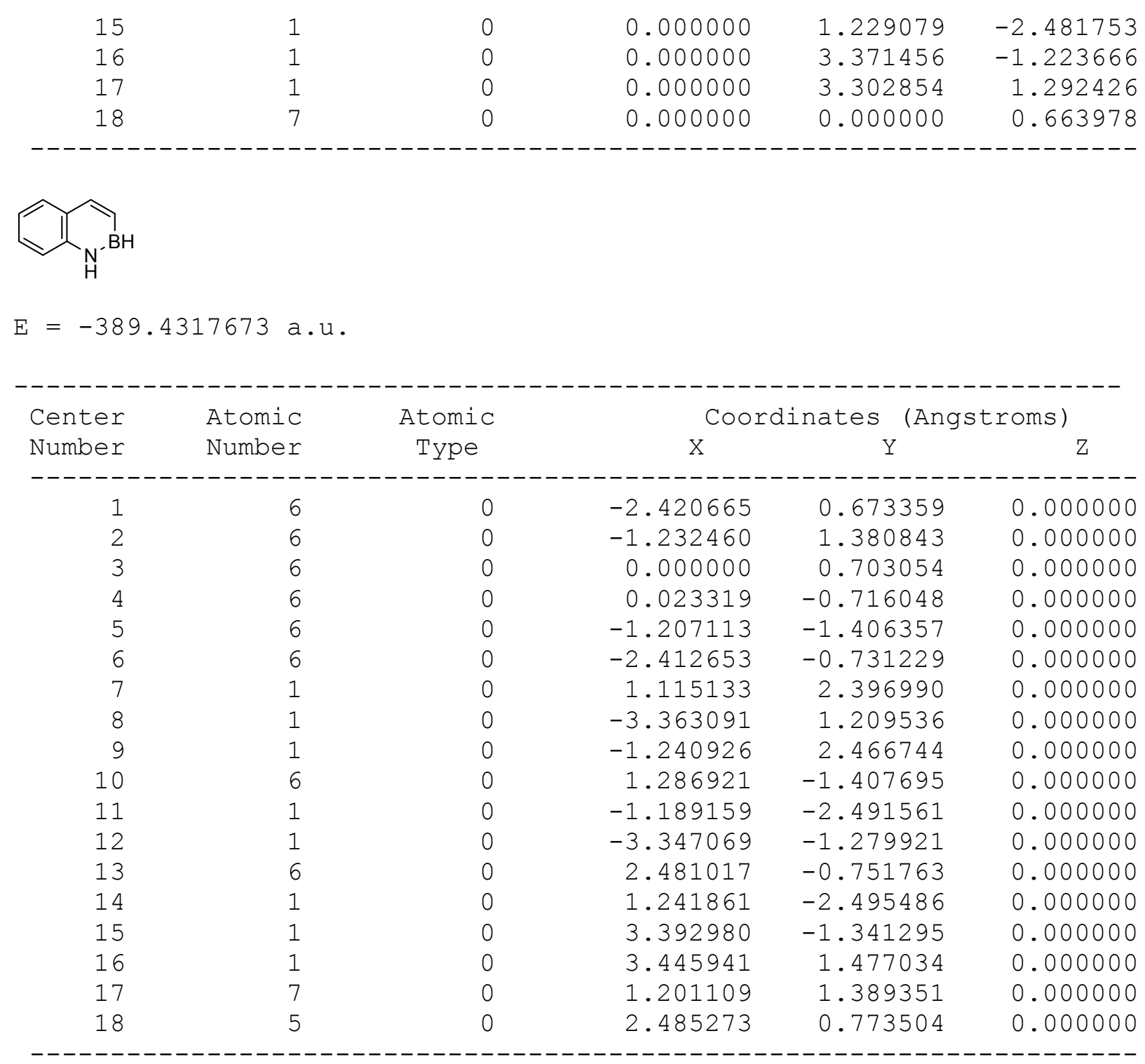

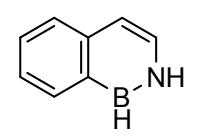

$E=-389.431359$ a.u.

\begin{tabular}{|c|c|c|c|c|c|}
\hline \multirow{2}{*}{$\begin{array}{l}\text { Center } \\
\text { Number }\end{array}$} & \multirow{2}{*}{$\begin{array}{l}\text { Atomic } \\
\text { Number }\end{array}$} & \multirow{2}{*}{$\begin{array}{l}\text { Atomic } \\
\text { Type }\end{array}$} & \multicolumn{3}{|c|}{ Coordinates (Angstroms) } \\
\hline & & & $\mathrm{X}$ & $\mathrm{Y}$ & z \\
\hline 1 & 6 & 0 & -2.516256 & -0.573938 & 0.000000 \\
\hline 2 & 6 & 0 & -1.362361 & -1.337720 & 0.000000 \\
\hline 3 & 6 & 0 & -0.083538 & -0.740679 & 0.000000 \\
\hline 4 & 6 & 0 & 0.000000 & 0.683256 & 0.000000 \\
\hline 5 & 6 & 0 & -1.187057 & 1.448697 & 0.000000 \\
\hline 6 & 6 & 0 & -2.422353 & 0.828619 & 0.000000 \\
\hline 7 & 1 & 0 & 1.285814 & -2.737935 & 0.000000 \\
\hline 8 & 1 & 0 & -3.490517 & -1.049878 & 0.000000 \\
\hline 9 & 1 & 0 & -1.433211 & -2.420980 & 0.000000 \\
\hline 10 & 6 & 0 & 1.294765 & 1.316226 & 0.000000 \\
\hline 11 & 1 & 0 & -1.121652 & 2.532214 & 0.000000 \\
\hline 12 & 1 & 0 & -3.326497 & 1.428159 & 0.000000 \\
\hline
\end{tabular}




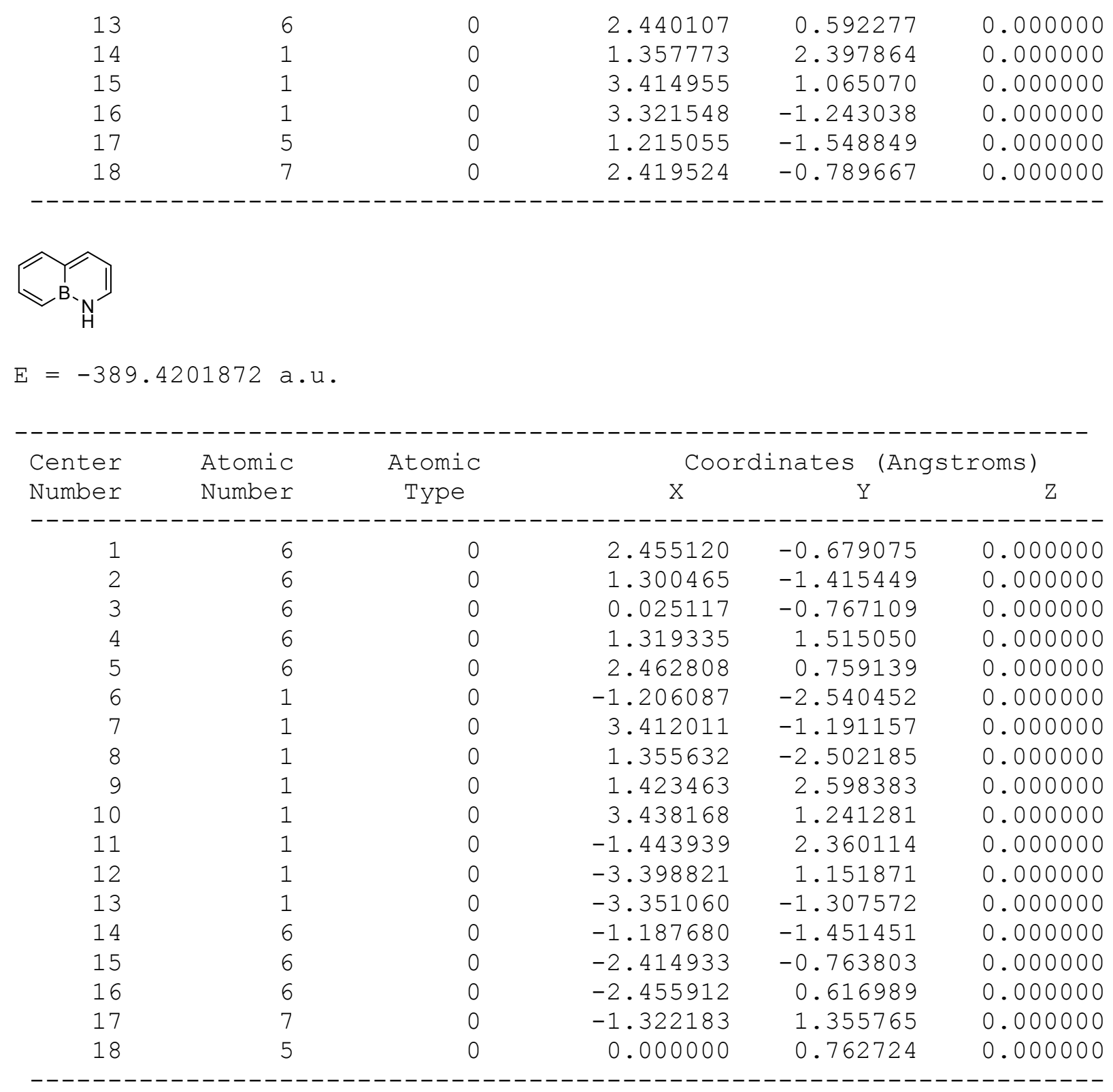

$\mathrm{C}_{\mathrm{B}}$

$E=-389.4023942$ a.u.

\begin{tabular}{|c|c|c|c|c|c|}
\hline \multirow{2}{*}{$\begin{array}{l}\text { Center } \\
\text { Number }\end{array}$} & \multirow{2}{*}{$\begin{array}{l}\text { Atomic } \\
\text { Number }\end{array}$} & \multirow{2}{*}{$\begin{array}{l}\text { Atomic } \\
\text { Type }\end{array}$} & \multicolumn{3}{|c|}{ Coordinates (Angstroms) } \\
\hline & & & $\mathrm{X}$ & $\mathrm{Y}$ & Z \\
\hline 1 & 6 & 0 & -2.436539 & 0.661833 & 0.000000 \\
\hline 2 & 6 & 0 & -1.267467 & 1.359547 & 0.000000 \\
\hline 3 & 6 & 0 & 0.000000 & 0.698239 & 0.000000 \\
\hline 4 & 6 & 0 & -1.190683 & -1.389223 & 0.000000 \\
\hline 5 & 6 & 0 & -2.393699 & -0.763270 & 0.000000 \\
\hline 6 & 1 & 0 & 1.157783 & 2.476839 & 0.000000 \\
\hline 7 & 1 & 0 & -3.388817 & 1.178454 & 0.000000 \\
\hline 8 & 1 & 0 & -1.261272 & 2.442806 & 0.000000 \\
\hline 9 & 1 & 0 & -1.091644 & -2.465587 & 0.000000 \\
\hline 10 & 1 & 0 & -3.301481 & -1.351913 & 0.000000 \\
\hline
\end{tabular}




$\begin{array}{rrrrrr}11 & 1 & 0 & 1.181345 & -2.639084 & 0.000000 \\ 12 & 1 & 0 & 3.519701 & -1.098748 & 0.000000 \\ 13 & 1 & 0 & 3.338969 & 1.350349 & 0.000000 \\ 14 & 6 & 0 & 1.205395 & 1.394906 & 0.000000 \\ 15 & 6 & 0 & 2.441131 & 0.736519 & 0.000000 \\ 16 & 6 & 0 & 2.532316 & -0.648320 & 0.000000 \\ 17 & 5 & 0 & 1.279560 & -1.452564 & 0.000000 \\ 18 & 7 & 0 & 0.014985 & -0.704525 & 0.000000 \\ -\end{array}$

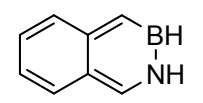

$E=-389.4146579$ a.u.

\begin{tabular}{|c|c|c|c|c|c|}
\hline \multirow{2}{*}{$\begin{array}{l}\text { Center } \\
\text { Number }\end{array}$} & \multirow{2}{*}{$\begin{array}{l}\text { Atomic } \\
\text { Number }\end{array}$} & \multirow{2}{*}{$\begin{array}{l}\text { Atomic } \\
\text { Type }\end{array}$} & \multicolumn{3}{|c|}{ Coordinates (Angstroms) } \\
\hline & & & $\mathrm{X}$ & $Y$ & Z \\
\hline 1 & 6 & 0 & -2.430968 & 0.687448 & 0.000000 \\
\hline 2 & 6 & 0 & -1.252919 & 1.374405 & 0.000000 \\
\hline 3 & 6 & 0 & 0.000000 & 0.681173 & 0.000000 \\
\hline 4 & 6 & 0 & 0.023533 & -0.770650 & 0.000000 \\
\hline 5 & 6 & 0 & -1.249035 & -1.438476 & 0.000000 \\
\hline 6 & 6 & 0 & -2.422295 & -0.741430 & 0.000000 \\
\hline 7 & 1 & 0 & 1.208713 & 2.466180 & 0.000000 \\
\hline 8 & 1 & 0 & -3.376246 & 1.217383 & 0.000000 \\
\hline 9 & 1 & 0 & -1.247372 & 2.459957 & 0.000000 \\
\hline 10 & 6 & 0 & 1.242122 & -1.454849 & 0.000000 \\
\hline 11 & 1 & 0 & -1.254763 & -2.523306 & 0.000000 \\
\hline 12 & 1 & 0 & -3.367116 & -1.274377 & 0.000000 \\
\hline 13 & 1 & 0 & 1.195820 & -2.540677 & 0.000000 \\
\hline 14 & 1 & 0 & 3.637465 & -1.140400 & 0.000000 \\
\hline 15 & 1 & 0 & 3.203072 & 1.336908 & 0.000000 \\
\hline 16 & 7 & 0 & 2.384889 & 0.742529 & 0.000000 \\
\hline 17 & 6 & 0 & 1.198364 & 1.381443 & 0.000000 \\
\hline 18 & 5 & 0 & 2.530679 & -0.702750 & 0.000000 \\
\hline
\end{tabular}

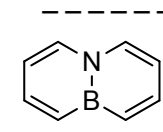

$E=-389.4116986 \mathrm{a} \cdot \mathrm{u}$.

\begin{tabular}{|c|c|c|c|c|c|}
\hline \multirow{2}{*}{$\begin{array}{l}\text { Center } \\
\text { Number }\end{array}$} & \multirow{2}{*}{$\begin{array}{l}\text { Atomic } \\
\text { Number }\end{array}$} & \multirow{2}{*}{$\begin{array}{l}\text { Atomic } \\
\text { Type }\end{array}$} & \multicolumn{3}{|c|}{ Coordinates (Angstroms) } \\
\hline & & & $\mathrm{X}$ & $\mathrm{Y}$ & Z \\
\hline 1 & 6 & 0 & 0.000000 & 2.494694 & -0.691241 \\
\hline 2 & 6 & 0 & 0.000000 & 1.371581 & -1.471210 \\
\hline 3 & 6 & 0 & 0.000000 & 1.190917 & 1.364146 \\
\hline 4 & 6 & 0 & 0.000000 & 2.396240 & 0.734491 \\
\hline 5 & 1 & 0 & 0.000000 & -1.491578 & -2.551231 \\
\hline 6 & 1 & 0 & 0.000000 & 3.489358 & -1.129399 \\
\hline 7 & 1 & 0 & 0.000000 & 1.491578 & -2.551231 \\
\hline 8 & 1 & 0 & 0.000000 & 1.108315 & 2.445117 \\
\hline 9 & 1 & 0 & 0.000000 & 3.292773 & 1.342323 \\
\hline 10 & 1 & 0 & 0.000000 & -1.108315 & 2.445117 \\
\hline 11 & 1 & 0 & 0.000000 & -3.292773 & 1.342323 \\
\hline
\end{tabular}




$\begin{array}{rrrrrr}12 & 1 & 0 & 0.000000 & -3.489358 & -1.129399 \\ 13 & 6 & 0 & 0.000000 & -1.371581 & -1.471210 \\ 14 & 6 & 0 & 0.000000 & -2.494694 & -0.691241 \\ 15 & 6 & 0 & 0.000000 & -2.396240 & 0.734491 \\ 16 & 7 & 0 & 0.000000 & 0.000000 & 0.661829 \\ 17 & 6 & 0 & 0.000000 & -1.190917 & 1.364146 \\ 18 & 5 & 0 & 0.000000 & 0.000000 & -0.816130 \\ - & & 0 & \end{array}$<smiles>c1ccc2[o+]c[nH]c2c1</smiles>

$E=-389.3763263$ a.u.

\begin{tabular}{|c|c|c|c|c|c|}
\hline \multirow{2}{*}{$\begin{array}{l}\text { Center } \\
\text { Number }\end{array}$} & \multirow{2}{*}{$\begin{array}{l}\text { Atomic } \\
\text { Number }\end{array}$} & \multirow{2}{*}{$\begin{array}{c}\text { Atomic } \\
\text { Type }\end{array}$} & \multicolumn{3}{|c|}{ Coordinates (Angstroms) } \\
\hline & & & $\mathrm{x}$ & $\mathrm{Y}$ & Z \\
\hline 1 & 6 & 0 & 2.411484 & -0.785498 & 0.000000 \\
\hline 2 & 6 & 0 & 1.279693 & 1.452368 & 0.000000 \\
\hline 3 & 1 & 0 & -1.218070 & -2.442030 & 0.000000 \\
\hline 4 & 1 & 0 & 3.209277 & -1.522978 & 0.000000 \\
\hline 5 & 1 & 0 & 1.106801 & -2.355261 & 0.000000 \\
\hline 6 & 1 & 0 & 1.213910 & 2.539318 & 0.000000 \\
\hline 7 & 1 & 0 & 3.657874 & 1.218621 & 0.000000 \\
\hline 8 & 1 & 0 & -1.221862 & 2.528212 & 0.000000 \\
\hline 9 & 1 & 0 & -3.349991 & 1.288444 & 0.000000 \\
\hline 10 & 1 & 0 & -3.352565 & -1.203102 & 0.000000 \\
\hline 11 & 6 & 0 & -1.224721 & -1.355780 & 0.000000 \\
\hline 12 & 6 & 0 & -2.412434 & -0.664459 & 0.000000 \\
\hline 13 & 6 & 0 & -2.407894 & 0.752114 & 0.000000 \\
\hline 14 & 6 & 0 & -1.223222 & 1.443569 & 0.000000 \\
\hline 15 & 6 & 0 & 0.000000 & -0.653001 & 0.000000 \\
\hline 16 & 6 & 0 & 0.039761 & 0.775491 & 0.000000 \\
\hline 17 & 7 & 0 & 1.184669 & -1.344959 & 0.000000 \\
\hline 18 & 5 & 0 & 2.577190 & 0.714931 & 0.000000 \\
\hline
\end{tabular}<smiles>c1ccc2cnccc2c1</smiles>

$E=-389.3773564$ a.u.

\begin{tabular}{|c|c|c|c|c|c|}
\hline \multirow{2}{*}{$\begin{array}{l}\text { Center } \\
\text { Number }\end{array}$} & \multirow{2}{*}{$\begin{array}{l}\text { Atomic } \\
\text { Number }\end{array}$} & \multirow{2}{*}{$\begin{array}{l}\text { Atomic } \\
\text { Type }\end{array}$} & \multicolumn{3}{|c|}{ Coordinates (Angstroms) } \\
\hline & & & $\mathrm{X}$ & $\mathrm{Y}$ & $\mathrm{Z}$ \\
\hline 1 & 6 & 0 & -2.481630 & 0.783534 & 0.000000 \\
\hline 2 & 6 & 0 & -1.255047 & -1.292725 & 0.000000 \\
\hline 3 & 1 & 0 & 1.435299 & 2.447564 & 0.000000 \\
\hline 4 & 1 & 0 & -3.505394 & 1.139802 & 0.000000 \\
\hline 5 & 1 & 0 & -1.240832 & 2.787299 & 0.000000 \\
\hline 6 & 1 & 0 & -1.357647 & -2.369570 & 0.000000 \\
\hline 7 & 1 & 0 & -3.251071 & -1.114701 & 0.000000 \\
\hline 8 & 1 & 0 & 1.125548 & -2.513282 & 0.000000 \\
\hline 9 & 1 & 0 & 3.326648 & -1.407090 & 0.000000 \\
\hline
\end{tabular}




\begin{tabular}{|c|c|c|c|c|c|}
\hline 10 & 1 & 0 & 3.481334 & 1.074923 & 0.000000 \\
\hline 11 & 6 & 0 & 1.360583 & 1.364929 & 0.000000 \\
\hline 12 & 6 & 0 & 2.506242 & 0.599946 & 0.000000 \\
\hline 13 & 6 & 0 & 2.419724 & -0.812402 & 0.000000 \\
\hline 14 & 6 & 0 & 1.193617 & -1.429675 & 0.000000 \\
\hline 15 & 6 & 0 & 0.066507 & 0.781169 & 0.000000 \\
\hline 16 & 6 & 0 & 0.000000 & -0.653284 & 0.000000 \\
\hline 17 & 5 & 0 & -1.228734 & 1.594165 & 0.000000 \\
\hline 18 & 7 & 0 & -2.390028 & -0.580675 & 0.000000 \\
\hline
\end{tabular}

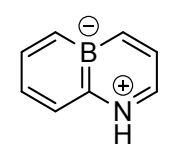

$E=-389.3805876 \mathrm{a} \cdot \mathrm{u}$.

\begin{tabular}{|c|c|c|c|c|c|}
\hline \multirow{2}{*}{$\begin{array}{l}\text { Center } \\
\text { Number }\end{array}$} & \multirow{2}{*}{$\begin{array}{l}\text { Atomic } \\
\text { Number }\end{array}$} & \multirow{2}{*}{$\begin{array}{c}\text { Atomic } \\
\text { Type }\end{array}$} & \multicolumn{3}{|c|}{ Coordinates (Angstroms) } \\
\hline & & & $\mathrm{X}$ & $Y$ & Z \\
\hline 1 & 6 & 0 & -2.402728 & 0.652512 & 0.000000 \\
\hline 2 & 6 & 0 & -1.319532 & -1.523133 & 0.000000 \\
\hline 3 & 1 & 0 & 1.155665 & 2.524254 & 0.000000 \\
\hline 4 & 1 & 0 & -3.284742 & 1.279026 & 0.000000 \\
\hline 5 & 1 & 0 & -1.241798 & 2.312545 & 0.000000 \\
\hline 6 & 1 & 0 & -1.461909 & -2.602219 & 0.000000 \\
\hline 7 & 1 & 0 & -3.463400 & -1.178355 & 0.000000 \\
\hline 8 & 1 & 0 & 1.619570 & -2.542280 & 0.000000 \\
\hline 9 & 1 & 0 & 3.536907 & -1.040758 & 0.000000 \\
\hline 10 & 1 & 0 & 3.312325 & 1.382828 & 0.000000 \\
\hline 11 & 6 & 0 & 1.188794 & 1.435451 & 0.000000 \\
\hline 12 & 6 & 0 & 2.404414 & 0.788047 & 0.000000 \\
\hline 13 & 6 & 0 & 2.527438 & -0.635829 & 0.000000 \\
\hline 14 & 6 & 0 & 1.435123 & -1.469748 & 0.000000 \\
\hline 15 & 6 & 0 & 0.000000 & 0.671331 & 0.000000 \\
\hline 16 & 6 & 0 & -2.470450 & -0.739469 & 0.000000 \\
\hline 17 & 7 & 0 & -1.219569 & 1.298168 & 0.000000 \\
\hline 18 & 5 & 0 & 0.037202 & -0.859438 & 0.000000 \\
\hline
\end{tabular}<smiles></smiles>

$E=-389.3622686 \mathrm{a} \cdot \mathrm{u}$.

\begin{tabular}{|c|c|c|c|c|c|}
\hline \multirow{2}{*}{$\begin{array}{l}\text { Center } \\
\text { Number }\end{array}$} & \multirow{2}{*}{$\begin{array}{l}\text { Atomic } \\
\text { Number }\end{array}$} & \multirow{2}{*}{$\begin{array}{c}\text { Atomic } \\
\text { Type }\end{array}$} & \multicolumn{3}{|c|}{ Coordinates (Angstroms) } \\
\hline & & & $\mathrm{x}$ & $\mathrm{Y}$ & Z \\
\hline 1 & 6 & 0 & 2.562154 & -0.658978 & 0.000000 \\
\hline 2 & 6 & 0 & 1.173258 & 1.349156 & 0.000000 \\
\hline 3 & 1 & 0 & -1.233896 & -2.495004 & 0.000000 \\
\hline 4 & 1 & 0 & 3.578395 & -1.043295 & 0.000000 \\
\hline 5 & 1 & 0 & 1.327695 & -2.709656 & 0.000000 \\
\hline 6 & 1 & 0 & 1.060172 & 2.423842 & 0.000000 \\
\hline 7 & 1 & 0 & 3.269908 & 1.392229 & 0.000000 \\
\hline
\end{tabular}




\begin{tabular}{|c|c|c|c|c|c|}
\hline 8 & 1 & 0 & -1.117407 & 2.406385 & 0.000000 \\
\hline 9 & 1 & 0 & -3.308899 & 1.274188 & 0.000000 \\
\hline 10 & 1 & 0 & -3.391267 & -1.253305 & 0.000000 \\
\hline 11 & 6 & 0 & -1.252493 & -1.411941 & 0.000000 \\
\hline 12 & 6 & 0 & -2.442906 & -0.731062 & 0.000000 \\
\hline 13 & 6 & 0 & -2.400978 & 0.684294 & 0.000000 \\
\hline 14 & 6 & 0 & -1.204724 & 1.328845 & 0.000000 \\
\hline 15 & 6 & 0 & 0.009298 & -0.769289 & 0.000000 \\
\hline 16 & 6 & 0 & 2.408452 & 0.729296 & 0.000000 \\
\hline 17 & 5 & 0 & 1.340586 & -1.515509 & 0.000000 \\
\hline 18 & 7 & 0 & 0.000000 & 0.637177 & 0.00000 \\
\hline
\end{tabular}

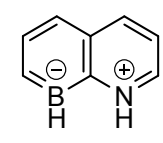

$E=-389.3656123 \mathrm{a} \cdot \mathrm{u}$.

\begin{tabular}{|c|c|c|c|c|c|}
\hline \multirow{2}{*}{$\begin{array}{l}\text { Center } \\
\text { Number }\end{array}$} & \multirow{2}{*}{$\begin{array}{l}\text { Atomic } \\
\text { Number }\end{array}$} & \multirow{2}{*}{$\begin{array}{l}\text { Atomic } \\
\text { Type }\end{array}$} & \multicolumn{3}{|c|}{ Coordinates (Angstroms) } \\
\hline & & & $\mathrm{X}$ & $\mathrm{Y}$ & Z \\
\hline 1 & 6 & 0 & -2.413644 & -0.619434 & 0.000000 \\
\hline 2 & 6 & 0 & -1.177882 & 1.431672 & 0.000000 \\
\hline 3 & 1 & 0 & 1.166811 & -2.771919 & 0.000000 \\
\hline 4 & 1 & 0 & -3.320649 & -1.208265 & 0.000000 \\
\hline 5 & 1 & 0 & -1.270366 & -2.296274 & 0.000000 \\
\hline 6 & 1 & 0 & -1.156090 & 2.517149 & 0.000000 \\
\hline 7 & 1 & 0 & -3.332672 & 1.317624 & 0.000000 \\
\hline 8 & 1 & 0 & 1.333414 & 2.452406 & 0.000000 \\
\hline 9 & 1 & 0 & 3.414642 & 1.185561 & 0.000000 \\
\hline 10 & 1 & 0 & 3.505933 & -1.232835 & 0.000000 \\
\hline 11 & 6 & 0 & 2.517626 & -0.778756 & 0.000000 \\
\hline 12 & 6 & 0 & 2.479793 & 0.628137 & 0.000000 \\
\hline 13 & 6 & 0 & 1.301323 & 1.368367 & 0.000000 \\
\hline 14 & 6 & 0 & 0.000000 & -0.719205 & 0.000000 \\
\hline 15 & 6 & 0 & -2.397016 & 0.774961 & 0.000000 \\
\hline 16 & 7 & 0 & -1.253081 & -1.282165 & 0.000000 \\
\hline 17 & 6 & 0 & 0.046287 & 0.727873 & 0.000000 \\
\hline 18 & 5 & 0 & 1.258323 & -1.573995 & 0.000000 \\
\hline
\end{tabular}

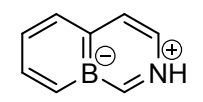

$E=-389.378468 \mathrm{a} \cdot \mathrm{u}$.

\begin{tabular}{|c|c|c|c|c|c|}
\hline \multirow{2}{*}{$\begin{array}{l}\text { Center } \\
\text { Number }\end{array}$} & \multirow{2}{*}{$\begin{array}{l}\text { Atomic } \\
\text { Number }\end{array}$} & \multirow{2}{*}{$\begin{array}{l}\text { Atomic } \\
\text { Type }\end{array}$} & \multicolumn{3}{|c|}{ Coordinates (Angstroms) } \\
\hline & & & $\mathrm{X}$ & $\mathrm{Y}$ & z \\
\hline 1 & 6 & 0 & -1.266791 & -1.362186 & 0.000000 \\
\hline 2 & 1 & 0 & 1.635523 & 2.507417 & 0.000000 \\
\hline 3 & 1 & 0 & -3.284872 & 1.217615 & 0.000000 \\
\hline 4 & 1 & 0 & -1.450260 & 2.557586 & 0.000000 \\
\hline 5 & 1 & 0 & -1.360710 & -2.445627 & 0.000000 \\
\hline 6 & 1 & 0 & -3.413673 & -1.065410 & 0.000000 \\
\hline
\end{tabular}




\begin{tabular}{|c|c|c|c|c|c|}
\hline 7 & 1 & 0 & 1.194978 & -2.563711 & 0.000000 \\
\hline 8 & 1 & 0 & 3.338871 & -1.412096 & 0.000000 \\
\hline 9 & 1 & 0 & 3.558214 & 1.009097 & 0.000000 \\
\hline 10 & 6 & 0 & 2.549345 & 0.601936 & 0.000000 \\
\hline 11 & 6 & 0 & 2.426507 & -0.823000 & 0.000000 \\
\hline 12 & 6 & 0 & 1.214184 & -1.475328 & 0.000000 \\
\hline 13 & 6 & 0 & -2.426427 & -0.625094 & 0.000000 \\
\hline 14 & 6 & 0 & 0.000000 & -0.736736 & 0.000000 \\
\hline 15 & 6 & 0 & 1.455811 & 1.433945 & 0.000000 \\
\hline 16 & 6 & 0 & -1.262001 & 1.487680 & 0.000000 \\
\hline 17 & 7 & 0 & -2.390096 & 0.744868 & 0.00000 \\
\hline 18 & 5 & 0 & 0.073767 & 0.794752 & 0.0000 \\
\hline
\end{tabular}

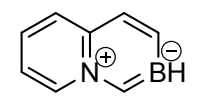

$\mathrm{E}=-389.3603588 \mathrm{a} \cdot \mathrm{u}$.

\begin{tabular}{|c|c|c|c|c|c|}
\hline \multirow{2}{*}{$\begin{array}{l}\text { Center } \\
\text { Number }\end{array}$} & \multirow{2}{*}{$\begin{array}{l}\text { Atomic } \\
\text { Number }\end{array}$} & \multirow{2}{*}{$\begin{array}{c}\text { Atomic } \\
\text { Type }\end{array}$} & \multicolumn{3}{|c|}{ Coordinates (Angstroms) } \\
\hline & & & $\mathrm{X}$ & $Y$ & Z \\
\hline 1 & 6 & 0 & 1.199547 & 1.451504 & 0.000000 \\
\hline 2 & 1 & 0 & -0.997722 & -2.490948 & 0.000000 \\
\hline 3 & 1 & 0 & 3.611147 & -1.227104 & 0.000000 \\
\hline 4 & 1 & 0 & 1.146081 & -2.436301 & 0.000000 \\
\hline 5 & 1 & 0 & 1.067982 & 2.530469 & 0.000000 \\
\hline 6 & 1 & 0 & 3.298530 & 1.553635 & 0.000000 \\
\hline 7 & 1 & 0 & -1.290212 & 2.400223 & 0.000000 \\
\hline 8 & 1 & 0 & -3.402090 & 1.086221 & 0.000000 \\
\hline 9 & 1 & 0 & -3.228651 & -1.439302 & 0.000000 \\
\hline 10 & 6 & 0 & -2.342942 & -0.816887 & 0.000000 \\
\hline 11 & 6 & 0 & -2.437588 & 0.595542 & 0.000000 \\
\hline 12 & 6 & 0 & -1.274024 & 1.316962 & 0.000000 \\
\hline 13 & 6 & 0 & 2.447140 & 0.876805 & 0.000000 \\
\hline 14 & 6 & 0 & 0.000000 & 0.701187 & 0.000000 \\
\hline 15 & 6 & 0 & -1.123910 & -1.417933 & 0.000000 \\
\hline 16 & 6 & 0 & 1.273541 & -1.358821 & 0.000000 \\
\hline 17 & 5 & 0 & 2.575088 & -0.636123 & 0.000000 \\
\hline 18 & 7 & 0 & 0.066988 & -0.698061 & 0.000000 \\
\hline
\end{tabular}<smiles>B1C=Cc2ccccc21</smiles>

$E=-389.395154 \mathrm{a} \cdot \mathrm{u}$.

\begin{tabular}{|c|c|c|c|c|c|}
\hline \multirow{2}{*}{$\begin{array}{l}\text { Center } \\
\text { Number }\end{array}$} & \multirow{2}{*}{$\begin{array}{l}\text { Atomic } \\
\text { Number }\end{array}$} & \multirow{2}{*}{$\begin{array}{l}\text { Atomic } \\
\text { Type }\end{array}$} & \multicolumn{3}{|c|}{ Coordinates (Angstroms) } \\
\hline & & & $\mathrm{x}$ & $Y$ & Z \\
\hline 1 & 1 & 0 & -1.403371 & -2.460732 & 0.000000 \\
\hline 2 & 1 & 0 & 3.536005 & -1.170216 & 0.000000 \\
\hline 3 & 1 & 0 & 1.252672 & -2.781594 & 0.000000 \\
\hline 4 & 1 & 0 & 1.242436 & 2.265514 & 0.000000 \\
\hline
\end{tabular}




\begin{tabular}{|c|c|c|c|c|c|}
\hline 5 & 1 & 0 & 3.290954 & 1.265863 & 0.000000 \\
\hline 6 & 1 & 0 & -1.105063 & 2.492191 & 0.000000 \\
\hline 7 & 1 & 0 & -3.307827 & 1.384193 & 0.000000 \\
\hline 8 & 1 & 0 & -3.466907 & -1.097319 & 0.000000 \\
\hline 9 & 6 & 0 & -2.494039 & -0.619322 & 0.000000 \\
\hline 10 & 6 & 0 & -2.404487 & 0.784212 & 0.000000 \\
\hline 11 & 6 & 0 & -1.172418 & 1.408072 & 0.000000 \\
\hline 12 & 6 & 0 & 2.431947 & 0.601367 & 0.000000 \\
\hline 13 & 6 & 0 & 0.000000 & 0.627163 & 0.000000 \\
\hline 14 & 6 & 0 & -1.338495 & -1.377600 & 0.000000 \\
\hline 15 & 6 & 0 & -0.051592 & -0.789166 & 0.000000 \\
\hline 16 & 6 & 0 & 2.530877 & -0.761520 & 0.000000 \\
\hline 17 & 7 & 0 & 1.234280 & 1.256859 & 0.000000 \\
\hline 18 & 5 & 0 & 1.262078 & -1.587031 & 0.000000 \\
\hline
\end{tabular}<smiles>B1C=CC=CN1</smiles>

$E=-389.3657927$ a.u.

\begin{tabular}{|c|c|c|c|c|c|}
\hline \multirow{2}{*}{$\begin{array}{l}\text { Center } \\
\text { Number }\end{array}$} & \multirow{2}{*}{$\begin{array}{l}\text { Atomic } \\
\text { Number }\end{array}$} & \multirow{2}{*}{$\begin{array}{l}\text { Atomic } \\
\text { Type }\end{array}$} & \multicolumn{3}{|c|}{ Coordinates (Angstroms) } \\
\hline & & & $\mathrm{X}$ & $\mathrm{Y}$ & z \\
\hline 1 & 1 & 0 & 1.479187 & -2.685592 & 0.000000 \\
\hline 2 & 1 & 0 & -3.348314 & -1.352572 & 0.000000 \\
\hline 3 & 1 & 0 & -1.147716 & -2.558410 & 0.000000 \\
\hline 4 & 1 & 0 & -1.294551 & 2.260961 & 0.000000 \\
\hline 5 & 1 & 0 & -3.323668 & 1.154367 & 0.000000 \\
\hline 6 & 1 & 0 & 1.074745 & 2.510651 & 0.000000 \\
\hline 7 & 1 & 0 & 3.278135 & 1.472324 & 0.000000 \\
\hline 8 & 1 & 0 & 3.639794 & -0.917704 & 0.000000 \\
\hline 9 & 6 & 0 & 2.609969 & -0.569365 & 0.000000 \\
\hline 10 & 6 & 0 & 2.416890 & 0.806015 & 0.000000 \\
\hline 11 & 6 & 0 & 1.147813 & 1.427220 & 0.000000 \\
\hline 12 & 6 & 0 & -2.417029 & 0.563048 & 0.000000 \\
\hline 13 & 6 & 0 & 0.000000 & 0.655269 & 0.000000 \\
\hline 14 & 6 & 0 & 0.044311 & -0.794890 & 0.000000 \\
\hline 15 & 6 & 0 & -2.411848 & -0.812183 & 0.000000 \\
\hline 16 & 7 & 0 & -1.258173 & 1.250216 & 0.000000 \\
\hline 17 & 6 & 0 & -1.167591 & -1.472876 & 0.000000 \\
\hline 18 & 5 & 0 & 1.422900 & -1.489791 & 0.000000 \\
\hline
\end{tabular}

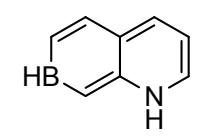

$E=-389.3691034$ a.u.

\begin{tabular}{cccccc} 
Center & Atomic & Atomic & \multicolumn{3}{c}{ Coordinates (Angstroms) } \\
Number & Number & Type & X & Y & $Z$ \\
-1 & 1 & 0 & 1.457747 & -2.402664 & 0.000000 \\
2 & 1 & 0 & -3.220615 & -1.515054 & 0.000000
\end{tabular}




\begin{tabular}{|c|c|c|c|c|c|}
\hline 3 & 1 & 0 & -0.945806 & -2.569361 & 0.000000 \\
\hline 4 & 1 & 0 & -1.403135 & 2.225676 & 0.000000 \\
\hline 5 & 1 & 0 & -3.363864 & 0.990889 & 0.000000 \\
\hline 6 & 1 & 0 & 0.893139 & 2.644762 & 0.000000 \\
\hline 7 & 1 & 0 & 3.459409 & 1.662810 & 0.000000 \\
\hline 8 & 1 & 0 & 3.504857 & -1.091917 & 0.000000 \\
\hline 9 & 6 & 0 & 2.556583 & -0.557987 & 0.000000 \\
\hline 10 & 6 & 0 & 1.091250 & 1.572916 & 0.000000 \\
\hline 11 & 6 & 0 & -2.421452 & 0.459198 & 0.000000 \\
\hline 12 & 6 & 0 & 0.000000 & 0.730094 & 0.000000 \\
\hline 13 & 6 & 0 & 0.113350 & -0.723032 & 0.000000 \\
\hline 14 & 6 & 0 & -2.325187 & -0.909876 & 0.000000 \\
\hline 15 & 7 & 0 & -1.305111 & 1.219317 & 0.000000 \\
\hline 16 & 6 & 0 & -1.040956 & -1.488075 & 0.000000 \\
\hline 17 & 6 & 0 & 1.421937 & -1.314711 & 0.000000 \\
\hline 18 & 5 & 0 & 2.476178 & 0.981695 & 0.000000 \\
\hline
\end{tabular}

(1)

$E=-389.3676053$ a.u.

\begin{tabular}{|c|c|c|c|c|c|}
\hline \multirow{2}{*}{$\begin{array}{l}\text { Center } \\
\text { Number }\end{array}$} & \multirow{2}{*}{$\begin{array}{l}\text { Atomic } \\
\text { Number }\end{array}$} & \multirow{2}{*}{$\begin{array}{l}\text { Atomic } \\
\text { Type }\end{array}$} & \multicolumn{3}{|c|}{ Coordinates (Angstroms) } \\
\hline & & & $\mathrm{X}$ & $\mathrm{Y}$ & z \\
\hline 1 & 1 & 0 & -1.396919 & 2.403580 & 0.000000 \\
\hline 2 & 1 & 0 & 3.307920 & 1.486052 & 0.000000 \\
\hline 3 & 1 & 0 & 1.117889 & 2.540597 & 0.000000 \\
\hline 4 & 1 & 0 & 1.476241 & -2.657469 & 0.000000 \\
\hline 5 & 1 & 0 & 3.652651 & -0.904652 & 0.000000 \\
\hline 6 & 1 & 0 & -1.151647 & -2.517447 & 0.000000 \\
\hline 7 & 1 & 0 & -3.169798 & -1.365041 & 0.000000 \\
\hline 8 & 1 & 0 & -3.429315 & 0.971826 & 0.000000 \\
\hline 9 & 6 & 0 & -2.426892 & 0.567902 & 0.000000 \\
\hline 10 & 6 & 0 & -1.122058 & -1.433946 & 0.000000 \\
\hline 11 & 6 & 0 & 2.625504 & -0.547781 & 0.000000 \\
\hline 12 & 6 & 0 & 0.067478 & -0.741727 & 0.000000 \\
\hline 13 & 6 & 0 & 0.000000 & 0.718881 & 0.000000 \\
\hline 14 & 6 & 0 & 2.440365 & 0.827564 & 0.000000 \\
\hline 15 & 6 & 0 & 1.173108 & 1.456728 & 0.000000 \\
\hline 16 & 6 & 0 & -1.301740 & 1.323854 & 0.000000 \\
\hline 17 & 5 & 0 & 1.428438 & -1.460314 & 0.000000 \\
\hline 18 & 7 & 0 & -2.326258 & -0.812104 & 0.000000 \\
\hline
\end{tabular}

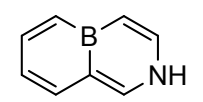

$E=-389.3845742$ a.u.

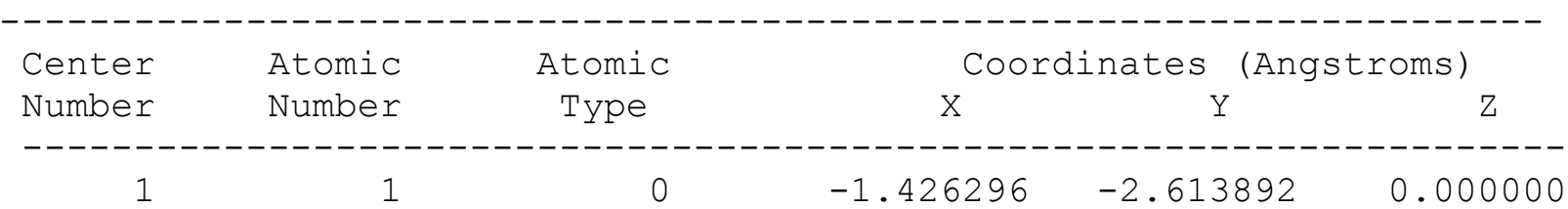




\begin{tabular}{|c|c|c|c|c|c|}
\hline 2 & 1 & 0 & 3.555317 & -1.002218 & 0.000000 \\
\hline 3 & 1 & 0 & 1.656856 & -2.512736 & 0.000000 \\
\hline 4 & 1 & 0 & 1.180608 & 2.560500 & 0.000000 \\
\hline 5 & 1 & 0 & 3.335500 & 1.422832 & 0.000000 \\
\hline 6 & 1 & 0 & -1.379544 & 2.398602 & 0.000000 \\
\hline 7 & 1 & 0 & -3.259499 & 1.092386 & 0.000000 \\
\hline 8 & 1 & 0 & -3.435084 & -1.189942 & 0.000000 \\
\hline 9 & 6 & 0 & -2.428493 & -0.786350 & 0.000000 \\
\hline 10 & 6 & 0 & -1.235922 & 1.321259 & 0.000000 \\
\hline 11 & 6 & 0 & 2.423271 & 0.834613 & 0.000000 \\
\hline 12 & 6 & 0 & 0.000000 & 0.710220 & 0.000000 \\
\hline 13 & 6 & 0 & 2.543613 & -0.600699 & 0.000000 \\
\hline 14 & 6 & 0 & 1.463344 & -1.441775 & 0.000000 \\
\hline 15 & 6 & 0 & -1.291389 & -1.535694 & 0.000000 \\
\hline 16 & 7 & 0 & -2.382680 & 0.593818 & 0.000000 \\
\hline 17 & 6 & 0 & 1.215726 & 1.472561 & 0.000000 \\
\hline 18 & 5 & 0 & 0.062000 & -0.831412 & 0.00000 \\
\hline
\end{tabular}

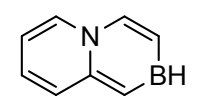

$E=-389.36597 \mathrm{a} \cdot \mathrm{u}$.

\begin{tabular}{|c|c|c|c|c|c|}
\hline \multirow{2}{*}{$\begin{array}{l}\text { Center } \\
\text { Number }\end{array}$} & \multirow{2}{*}{$\begin{array}{l}\text { Atomic } \\
\text { Number }\end{array}$} & \multirow{2}{*}{$\begin{array}{c}\text { Atomic } \\
\text { Type }\end{array}$} & \multicolumn{3}{|c|}{ Coordinates (Angstroms) } \\
\hline & & & $\mathrm{X}$ & $Y$ & Z \\
\hline 1 & 1 & 0 & 0.935325 & 2.491899 & 0.000000 \\
\hline 2 & 1 & 0 & -3.336977 & 1.129587 & 0.000000 \\
\hline 3 & 1 & 0 & -1.193298 & 2.355874 & 0.000000 \\
\hline 4 & 1 & 0 & -1.100604 & -2.553305 & 0.000000 \\
\hline 5 & 1 & 0 & -3.288999 & -1.406107 & 0.000000 \\
\hline 6 & 1 & 0 & 1.280860 & -2.472721 & 0.000000 \\
\hline 7 & 1 & 0 & 3.686929 & -1.109615 & 0.000000 \\
\hline 8 & 1 & 0 & 3.208029 & 1.638292 & 0.000000 \\
\hline 9 & 6 & 0 & 2.401810 & 0.909941 & 0.000000 \\
\hline 10 & 6 & 0 & 1.325053 & -1.386852 & 0.000000 \\
\hline 11 & 6 & 0 & -2.365569 & -0.839782 & 0.000000 \\
\hline 12 & 6 & 0 & 0.087854 & -0.764079 & 0.000000 \\
\hline 13 & 6 & 0 & -2.401046 & 0.587473 & 0.000000 \\
\hline 14 & 6 & 0 & -1.235991 & 1.275420 & 0.000000 \\
\hline 15 & 6 & 0 & 1.147799 & 1.428208 & 0.000000 \\
\hline 16 & 6 & 0 & -1.163412 & -1.472200 & 0.000000 \\
\hline 17 & 5 & 0 & 2.605950 & -0.603308 & 0.000000 \\
\hline 18 & 7 & 0 & 0.000000 & 0.644837 & 0.000000 \\
\hline
\end{tabular}

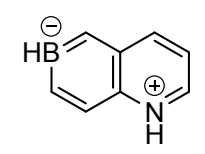

$E=-389.3562329 \mathrm{a} \cdot \mathrm{u}$.

$\begin{array}{llll}\text { Center } & \text { Atomic } & \text { Atomic } & \text { Coordinates (Angstroms) } \\ \text { Number } & \text { Number } & \text { Type } & \mathrm{X}\end{array}$




\begin{tabular}{|c|c|c|c|c|c|}
\hline 1 & 1 & 0 & 0.941828 & 2.587054 & 0.000000 \\
\hline 2 & 1 & 0 & -3.325739 & 1.049943 & 0.000000 \\
\hline 3 & 1 & 0 & -1.318525 & 2.203147 & 0.000000 \\
\hline 4 & 1 & 0 & -1.036626 & -2.602529 & 0.000000 \\
\hline 5 & 1 & 0 & -3.248427 & -1.478433 & 0.00000 \\
\hline 6 & 1 & 0 & 1.413357 & -2.466847 & 0.00000 \\
\hline 7 & 1 & 0 & 3.740308 & -0.986529 & 0.00000 \\
\hline 8 & 1 & 0 & 3.210453 & 1.707426 & 0.00000 \\
\hline 9 & 6 & 0 & 2.394030 & 0.987176 & 0.00000 \\
\hline 10 & 6 & 0 & 1.399199 & -1.378283 & 0.00000 \\
\hline 11 & 6 & 0 & -2.332416 & -0.902759 & 0.00000 \\
\hline 12 & 6 & 0 & 0.126798 & -0.791597 & 0.00000 \\
\hline 13 & 6 & 0 & -2.403436 & 0.487397 & 0.00000 \\
\hline 14 & 6 & 0 & 1.121487 & 1.511475 & 0.00000 \\
\hline 15 & 6 & 0 & -1.093622 & -1.518996 & 0.00000 \\
\hline 16 & 5 & 0 & 2.633318 & -0.532097 & 0.00000 \\
\hline 17 & 6 & 0 & 0.000000 & 0.656504 & 0.00000 \\
\hline 18 & 7 & 0 & -1.259351 & 1.191679 & 0.000000 \\
\hline
\end{tabular}<smiles>b1ccc2ccccc2c1</smiles>

$E=-389.355044$ a.u.

\begin{tabular}{|c|c|c|c|c|c|}
\hline \multirow{2}{*}{$\begin{array}{l}\text { Center } \\
\text { Number }\end{array}$} & \multirow{2}{*}{$\begin{array}{l}\text { Atomic } \\
\text { Number }\end{array}$} & \multirow{2}{*}{$\begin{array}{c}\text { Atomic } \\
\text { Type }\end{array}$} & \multicolumn{3}{|c|}{ Coordinates (Angstroms) } \\
\hline & & & $\mathrm{X}$ & $\mathrm{Y}$ & z \\
\hline 1 & 1 & 0 & -1.135274 & -2.573863 & 0.000000 \\
\hline 2 & 1 & 0 & 3.648025 & -0.862519 & 0.000000 \\
\hline 3 & 1 & 0 & 1.536427 & -2.657496 & 0.000000 \\
\hline 4 & 1 & 0 & 1.071263 & 2.546267 & 0.000000 \\
\hline 5 & 1 & 0 & 3.287978 & 1.531154 & 0.000000 \\
\hline 6 & 1 & 0 & -1.402318 & 2.338896 & 0.000000 \\
\hline 7 & 1 & 0 & -3.272363 & 0.995503 & 0.000000 \\
\hline 8 & 1 & 0 & -3.327293 & -1.344395 & 0.000000 \\
\hline 9 & 6 & 0 & -2.365028 & -0.854597 & 0.000000 \\
\hline 10 & 6 & 0 & -1.263606 & 1.264228 & 0.000000 \\
\hline 11 & 6 & 0 & 2.423649 & 0.869332 & 0.000000 \\
\hline 12 & 6 & 0 & 0.000000 & 0.669613 & 0.000000 \\
\hline 13 & 6 & 0 & 2.614355 & -0.522121 & 0.000000 \\
\hline 14 & 6 & 0 & -1.150216 & -1.489756 & 0.000000 \\
\hline 15 & 6 & 0 & 1.166870 & 1.465328 & 0.000000 \\
\hline 16 & 6 & 0 & 0.076563 & -0.788695 & 0.000000 \\
\hline 17 & 7 & 0 & -2.379489 & 0.522585 & 0.000000 \\
\hline 18 & 5 & 0 & 1.446892 & -1.462327 & 0.000000 \\
\hline
\end{tabular}

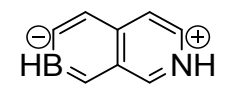

$E=-389.35401$ a.u.

$\begin{array}{llll}\text { Center } & \text { Atomic } & \text { Atomic } & \text { Coordinates (Angstroms) } \\ \text { Number } & \text { Number } & \text { Type } & \mathrm{X}\end{array}$




\begin{tabular}{|c|c|c|c|c|c|}
\hline 1 & 1 & 0 & -1.414837 & 2.354832 & 0.000000 \\
\hline 2 & 1 & 0 & 3.254945 & 1.691166 & 0.000000 \\
\hline 3 & 1 & 0 & 1.012576 & 2.611789 & 0.000000 \\
\hline 4 & 1 & 0 & 1.392094 & -2.448725 & 0.000000 \\
\hline 5 & 1 & 0 & 3.745871 & -1.009009 & 0.000000 \\
\hline 6 & 1 & 0 & -1.057847 & -2.549962 & 0.000000 \\
\hline 7 & 1 & 0 & -3.081915 & -1.454374 & 0.000000 \\
\hline 8 & 1 & 0 & -3.433881 & 0.860002 & 0.000000 \\
\hline 9 & 6 & 0 & -2.419553 & 0.491681 & 0.000000 \\
\hline 10 & 6 & 0 & -1.055576 & -1.467157 & 0.000000 \\
\hline 11 & 6 & 0 & 0.141786 & -0.733569 & 0.000000 \\
\hline 12 & 6 & 0 & 2.425673 & 0.984787 & 0.000000 \\
\hline 13 & 6 & 0 & -1.295784 & 1.277455 & 0.000000 \\
\hline 14 & 6 & 0 & 1.399519 & -1.359375 & 0.000000 \\
\hline 15 & 6 & 0 & 0.000000 & 0.724060 & 0.000000 \\
\hline 16 & 7 & 0 & -2.256696 & -0.871033 & 0.000000 \\
\hline 17 & 6 & 0 & 1.161038 & 1.533147 & 0.000000 \\
\hline 18 & 5 & 0 & 2.647449 & -0.532933 & 0.000000 \\
\hline
\end{tabular}

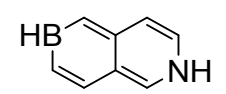

$E=-389.3640394$ a.u.

\begin{tabular}{|c|c|c|c|c|c|}
\hline \multirow{2}{*}{$\begin{array}{l}\text { Center } \\
\text { Number }\end{array}$} & \multirow{2}{*}{$\begin{array}{l}\text { Atomic } \\
\text { Number }\end{array}$} & \multirow{2}{*}{$\begin{array}{c}\text { Atomic } \\
\text { Type }\end{array}$} & \multicolumn{3}{|c|}{ Coordinates (Angstroms) } \\
\hline & & & $\mathrm{x}$ & $\mathrm{Y}$ & Z \\
\hline 1 & 1 & 0 & -1.031469 & -2.612494 & 0.000000 \\
\hline 2 & 1 & 0 & 3.751807 & -0.960026 & 0.000000 \\
\hline 3 & 1 & 0 & 1.436335 & -2.444306 & 0.000000 \\
\hline 4 & 1 & 0 & 0.969007 & 2.610903 & 0.000000 \\
\hline 5 & 1 & 0 & 3.239091 & 1.745651 & 0.000000 \\
\hline 6 & 1 & 0 & -1.427917 & 2.294099 & 0.000000 \\
\hline 7 & 1 & 0 & -3.284049 & 0.888781 & 0.000000 \\
\hline 8 & 1 & 0 & -3.232974 & -1.463102 & 0.000000 \\
\hline 9 & 6 & 0 & -2.292998 & -0.929201 & 0.000000 \\
\hline 10 & 6 & 0 & -1.263159 & 1.223338 & 0.000000 \\
\hline 11 & 6 & 0 & 0.000000 & 0.681124 & 0.000000 \\
\hline 12 & 6 & 0 & -1.078763 & -1.530012 & 0.000000 \\
\hline 13 & 6 & 0 & 1.154431 & 1.537294 & 0.000000 \\
\hline 14 & 6 & 0 & 0.150067 & -0.777373 & 0.000000 \\
\hline 15 & 7 & 0 & -2.377291 & 0.448679 & 0.000000 \\
\hline 16 & 6 & 0 & 1.406807 & -1.355630 & 0.000000 \\
\hline 17 & 6 & 0 & 2.419669 & 1.029010 & 0.000000 \\
\hline 18 & 5 & 0 & 2.648977 & -0.494313 & 0.000000 \\
\hline
\end{tabular}

